\title{
Les modèles \\ explicatifs \\ du cancer
}

\section{Aspects historiques}

> Les modèles explicatifs du cancer ont évolué en relation étroite avec les connaissances biologiques et leurs transformations. La situation n'est pas différente aujourd'hui. Les nouvelles techniques, comme le séquençage à haut débit, sont en train de transformer, d'une manière encore imprévisible, notre conception du cancer. La biologie des systèmes, l'épigénétique et les travaux sur les cellules souches renouvellent la vision du cancer et de son évolution. De nouveaux rôles sont donnés aux phénomènes aléatoires. Dans l'opposition traditionnelle dans la pensée biologique entre holisme et réductionnisme, c'est à un niveau intermédiaire, celui de la cellule, que l'étude de cette maladie apparaît la plus pertinente. <

«La biologie et la recherche sur le cancer se sont développées de concert. De manière invariable, à chaque étape, les caractéristiques de la cellule cancéreuse ont été attribuées à quelque défaut dans la branche de la biologie qui apparaissait à ce moment là passionnante et à la mode. » Cette phrase de John Cairns, tirée d'un ouvrage de 1978 [1], reflète bien l'histoire des recherches sur le cancer comme nous le montrerons dans la première partie de cet article. II ne faudrait pas en déduire pour autant que l'état des connaissances détermine la nature des modèles explicatifs du cancer. Nous verrons dans la deuxième partie que la théorie des mutations somatiques, aujourd'hui dominante, n'est pas le seul modèle qui ait été proposé lorsque la biologie moléculaire s'est imposée au début des années 1960. De même, nous mettrons en avant dans la troisième partie deux caractéristiques du cancer - être une maladie cellulaire et le fruit d'un processus évolutif - dont la prise en compte est requise pour mieux apprécier le rôle du hasard, du réductionnisme et du holisme dans l'explication du phénomène « cancer » (quatrième partie).

Les six articles publiés dans ce numéro de médecine/sciences regroupent des interventions faites dans le cadre d'un colloque intitulé «La recherche sur le cancer : un champ privilégié pour penser les rapports entre hasard, rédutionnisme et holisme », organisé le 7 novembre 2013, ENS-Paris, 45, rue d'Ulm, 75005 Paris, par le groupe de travail HAREDHOL (hasard, réductionnisme, holisme).

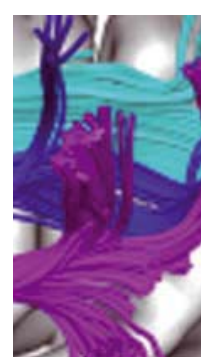

Centre Cavaillès, République des savoirs, USR 3608, École normale supérieure, 46, rue d'Ulm, 75005 Paris, France. morange@biologie.ens.fr

\section{Histoire de la biologie et histoire des théories sur le cancer}

Chaque avancée de la biologie a très rapidement conduit à la genèse d'une nouvelle «théorie du cancer». La mise en place de la théorie cellulaire amène Rudolf Virchow à proposer en 1858 la notion de maladie cellulaire dont lui et ses collaborateurs montreront rapidement que le cancer en est la meilleure illustration. En quelques années sont réunies les différentes formes de cancers, tumeurs solides, leucémies, lymphomes. Les caractéristiques des cellules cancéreuses et la modification de ces caractéristiques avec l'évolution de la maladie sont décrites; en découle une méthode de diagnostic, et en partie de pronostic. Est découvert aussi le phénomène de migration des cellules hors de la tumeur d'origine, suivi de la formation de métastases.

Quelques années plus tard, de manière indépendante et en partie antagoniste, la description du rôle des microorganismes dans la genèse des maladies infectieuses conduit de nombreux biologistes à rechercher, parfois avec des succès illusoires, les «microbes du cancer ». Après la découverte des virus filtrants, cette recherche, jusqu'alors peu fructueuse, laisse à partir de 1903 et du travail d'Amédée Borrel la place à celle, bien plus riche d'avenir, des virus du cancer. La place croissante que prennent, à partir de la redécouverte des lois de Mendel en 1900, les gènes dans les explications des biologistes amène progressivement ces derniers à envisager que des mutations soient à l'origine des cancers. Cette hypothèse sera lentement confortée par la caractérisation des substances cancérigènes présentes dans l'environnement et la démonstration de leurs effets mutagènes. De manière indépendante, Theodor Boveri propose en 1914 que le cancer trouve son origine dans une mauvaise répartition des chromosomes lors de la division cellulaire [2]. Les progrès de la biochimie conduisent Otto Warburg à faire I'hypothèse, dès 1915 , que le cancer est le résultat d'une perturbation 
des mécanismes d'oxydation cellulaire, hypothèse à laquelle il restera fidèle jusqu'à la fin de sa vie [3].

Si Cairns a raison, il ne faut pas oublier que ce parallélisme entre les transformations de la biologie et les nouvelles théories explicatives du cancer trouve aussi son origine dans les développements techniques et technologiques qui ont accompagné ces progrès et ouvert aux chercheurs de nouvelles approches expérimentales du cancer. Ce fut le cas avec la mise au point des outils du génie génétique qui a suivi l'essor de la biologie moléculaire, de même que le renouveau actuel d'interrogations sur le cancer est largement le résultat de la mise au point de nouvelles techniques de séquençage plus puissantes, plus rapides, et moins coûteuses. II devient possible de séquencer entièrement le génome de cellules tumorales à différents stades de l'évolution du cancer, et de comparer ces séquences à la séquence des tissus sains ; ou, à l'intérieur d'une tumeur, de comparer les séquences des différentes cellules qui la constituent [4].

\section{L'état des connaissances biologiques ne détermine pas le type de modèle proposé pour expliquer le cancer}

Le parallélisme décrit précédemment n'implique pas que les théories explicatives du cancer soient la conséquence directe de l'évolution des connaissances en biologie. Cela est particulièrement vrai du modèle explicatif qui se met en place entre 1975 et 1985 et domine alors le paysage explicatif du cancer, que l'on appelle de manière peu précise «la théorie des mutations somatiques ».

L'étude historique montre qu'au moins un autre modèle moléculaire a été proposé et a reçu un accueil favorable entre 1960 et 1980 [5]. II était la conséquence de l'intérêt suscité chez les biologistes par les premiers modèles de régulation génétique apparus au début des années 1960. Il visait aussi à expliquer pourquoi les cellules cancéreuses expriment souvent des caractères visibles à l'état embryonnaire, mais absents à l'état adulte. Le cancer était vu comme le résultat d'une perturbation des mécanismes régulateurs contrôlant la différenciation cellulaire et le développement embryonnaire. Le modèle qui s'est imposé en s'opposant au modèle précédent fit du cancer la conséquence d'une altération des voies de signalisation et des mécanismes qui contrôlent la division cellulaire. La surprise fut grande lorsqu'on démontra que la mutation du premier oncogène connu, ras, modifie la structure de la protéine codée par le gène, et non les séquences qui en contrôlent l'expression. La théorie actuelle ne dit pas seulement que le cancer est le résultat de mutations : elle précise la nature de ces mutations, les classes en différentes catégories [6], et explique pourquoi ces mutations donnent à la cellule cancéreuse ses propriétés particulières $(\rightarrow)[16] . \quad(\rightarrow)$ Voir l'article

de $\varepsilon$. Solary, page 683

\section{Deux caractéristiques propres au cancer}

de ce numéro

Le cancer reste une maladie cellulaire plus de 150 ans après l'introduction de cette hypothèse. Cela ne signifie pas simplement que les cellules sont affectées par le cancer : les cellules étant les éléments de base des organismes, toute maladie affecte, d'une manière ou d'une autre, les cellules. Cela signifie que la cause du cancer est d'une certaine manière interne aux cellules, et non externe; à la différence, par exemple, d'une maladie microbienne ou virale. II y a plusieurs conséquences, pour le cancer et son étude, du fait d'être une maladie cellulaire. La première est que le lignage des cellules concernées, le chemin qu'elles ont suivi dans leur transformation, leur «histoire», sont importants. La deuxième est que le cancer a à voir avec les cellules en tant qu'entités vivantes et en partie autonomes, et en particulier avec les relations que ces cellules entretiennent avec leur environnement. Ce qui a conduit dès la fin du $X I x^{e}$ siècle certains biologistes à interpréter le cancer comme une révolte des cellules contre l'organisme qui les porte $(\rightarrow)$ [17].

Cette dimension cellulaire du cancer est particulièrement visible dans la théorie des cellules $(\rightarrow)$ Voir l'article de C. Sonnenscheim et A.M. Soto, page 688 de ce numéro souches du cancer. Cette théorie, aujourd'hui très en vogue, est parfaitement compatible avec la théorie des mutations somatiques, mais la nature de la population de cellules qui est touchée par les mutations explique certaines caractéristiques des tumeurs - comme leur hétérogénéité - et oriente aussi différemment les stratégies de lutte contre le cancer.

Le cancer est aussi le résultat d'un processus d'évolution darwinienne au niveau cellulaire, avec mutations aléatoires et sélection des cellules qui se multiplient le plus rapidement dans un environnement donné [7]. La vision évolutive du cancer n'est pas nouvelle. En 1976, Peter Nowell avait proposé que les tumeurs soient le fruit d'une sélection clonale [8]. Cette vision évolutive a été préparée par la mise au point de modèles expérimentaux du cancer au milieu du $x x^{e}$ siècle, et par l'importance prise par la notion de progression mise en avant par Leslie Foulds dans les années $1950[9,10]$. Lorsque Bert Vogelstein décrit en 1996 la succession des mutations nécessaire pour engendrer un cancer colorectal [11], il procède à la traduction en termes génétiques et moléculaires de cette notion de progression.

Le modèle de Vogelstein et celui de Nowell reposent sur deux conceptions différentes de la notion d'évolution. Dans la première, il s'agit d'une évolution dirigée, orientée; dans la seconde, d'une évolution aléatoire, conduite par la seule sélection naturelle.

Le retour actuel d'une vision évolutive du cancer est aussi associé à une nouvelle conception de l'évolution. Le rôle des mutations dites neutres, car non triées par la sélection naturelle, y est valorisé. Une place pour des événements catastrophiques dans la genèse d'un cancer n'est pas non plus écartée [12] ; de même que l'existence de contraintes fortes guidant l'évolution des tumeurs 
est acceptée. Dans un tel modèle évolutif du cancer, l'explication de sa genèse et de son développement est multiple : elle est à chercher dans des processus aléatoires, dans la nature des mutations, aussi bien que dans l'action de la sélection naturelle. Le cancer, comme tout phénomène « historique», est le résultat d'une pluralité de causes.

\section{Le cancer, un domaine privilégié pour préciser les rapports entre hasard, réductionnisme et holisme?}

Parmi les domaines de recherche les plus actifs aujourd'hui en biologie, on peut citer, entre autres, la biologie des systèmes, l'épigénétique et, dans une moindre mesure, l'étude de l'expression stochastique des gènes. La biologie des systèmes est souvent considérée comme une approche holiste des phénomènes du vivant, et opposée à l'approche réductionniste de la biologie moléculaire qui l'a précédée. Les spécialistes de l'épigénétique mettent en avant le rôle de signaux extérieurs qui peuvent modifier les marques épigénétiques, et en conséquence l'activité des gènes. Sans être anti-réductionniste, l'épigénétique met l'accent sur les relations des organismes avec leur environnement. Enfin, par une étude très précise de la dynamique des processus se produisant au sein des cellules vivantes, le hasard s'introduit naturellement au cœur des processus biologiques $(\rightarrow)$ [18]. Autrement dit, la journée qui a donné naissance aux articles ici rassemblés échappe moins que tout $\rightarrow$ Voir l'article de J.P. Capp, page 693 de ce numéro autre à la règle que Cairns avait pointée du doigt.

Il est néanmoins nécessaire d'introduire dans le paysage les deux caractéristiques du cancer que nous avons décrites plus haut. Le fait que le cancer ait été, et soit encore, considéré comme une maladie cellulaire rend l'opposition holisme/réductionnisme moins pertinente. Un troisième acteur se glisse entre l'organisme et les gènes: la cellule. Faire de cette dernière le principal niveau d'étude du phénomène cancéreux sera considéré comme une attitude holiste par ceux qui ne prêtent d'importance qu'aux mutations des gènes, et réductionniste par ceux qui considèrent que la désorganisation des tissus de l'organisme est à l'origine du cancer. De nombreux travaux ont souligné ces dernières années le rôle de l'environnement interne, aussi bien dans la croissance des tumeurs que dans le développement des métastases. La notion de niche est de plus en plus utilisée pour désigner ce microenvironnement favorable à la croissance de la tumeur ou à la formation de métastases, comme elle l'est, et non sans lien, dans le cas des cellules souches. Le rôle de cet environnement peut être vu de manière «classique », comme intervenant dans le tri des mutations se produisant dans les cellules cancéreuses. Il peut être imaginé, de manière moins traditionnelle, comme modifiant le taux de mutation des cellules tumorales; ou comme créant des épimutations (par modifications épigénétiques) dont les effets peuvent être similaires à ceux des mutations. Comme noté précédemment, l'attention portée aux interactions entre les cellules cancéreuses et les cellules qui les entourent rend quelque peu obsolète la distinction holisme/réductionnisme ${ }^{1}$.

${ }^{1}$ Voir le numéro de médecine/sciences «Microenvironnements tumoraux : conflictuels et complémentaires », publié en avril 2014 ( $\mathrm{m} / \mathrm{s} 2014$, vol. $\left.30, \mathrm{n}^{\circ} 4\right)$.
Le hasard est celui des mutations, mais aussi celui qui accompagne les processus cellulaires lorsque ces derniers sont considérés à une échelle suffisamment fine. La question qui se pose est de savoir si cette deuxième forme de hasard joue un rôle particulier dans la genèse des tumeurs. C'est aussi le hasard qui explique la rétention (ou non) de mutations initialement neutres, qui peuvent ultérieurement contribuer au phénotype cancéreux.

\section{Conclusion}

Le séquençage des tumeurs avait pour objectif principal de «faire le tour » des gènes dont les mutations sont responsables des tumeurs. Décrire de nouveaux gènes impliqués dans la cancérogenèse, et donc de nouvelles cibles pour la thérapeutique, choisir, pour chaque tumeur, les meilleurs agents thérapeutiques ont été les motivations de ces projets $[13,14]$. Mais les résultats ont apporté plus. Ils ont démontré l'hétérogénéité cellulaire des tumeurs, et donc conforté la vision évolutive du cancer. Ils révèlent de nouveaux mécanismes d'oncogenèse liant par exemple l'altération du métabolisme à la modification de marques épigénétiques [15]. Ils étendent le rôle du hasard dans la genèse des tumeurs. Ils contribuent à faire, plus que jamais, de la cellule le cœur du processus cancéreux et le niveau privilégié où doit en être recherchée l'explication. $\diamond$

\section{SUMMARY}

Hypotheses for the genesis of cancer:

a historical perspective

The explanation of cancer has always been tightly related to the state of knowledge in biology, and its transformations. The present situation is not different. New techniques, such as deep sequencing, are rapidly moving our vision of cancer in an impredictable way. Systems biology, epigenetics, and the study of stem cells are generating new hypotheses on cancer and its evolution. New roles for aleatory events in the genesis of cancer have been proposed. In the traditional opposition between holism and reductionism, organisms and molecules, an intermediary level, the cancer cell, seems to be the most appropriate to study oncogenesis. $\diamond$

\section{LIENS D'INTÉRÊT}

L'auteur déclare n'avoir aucun lien d'intérêt concernant les données publiées dans cet article.

\section{RÉFÉRENCES}

1. Cairns J. Cancer: science and society. San Francisco : Freeman and Co, 1978 : $64 \mathrm{p}$.

2. Boveri T. The origin of malignant tumours. London : Baillière, Tindall and Cox, 1927. 


\section{RÉFÉRENCES}

3. Warburg 0. On the origin of cancer cells. Science $1956 ; 123: 309-14$.

4. Gerlinger M, Rowan AJ, Horswell S, et al. Intratumor heterogeneity and branched evolution revealed by multiregion sequencing. $N$ Engl J Med 2012 ; 366 : 883-92.

5. Morange M. From the regulatory vision of cancer to the oncogene paradigm, 1975-1985. J Hist Biol $1997 ; 30: 1-29$

6. Hanahan D, Weinberg RA. Hallmarks of cancer: the next generation. Cell $2011 ; 144: 646-74$.

7. Merlo RM, Pepper JW, Reid BJ, Maley CC. Cancer as an evolutionary and ecological process. Nat Rev Cancer $2006 ; 6: 924-35$.

8. Nowell PC. The clonal evolution of tumor cell populations. Science $1976 ; 194: 23-8$.

9. Foulds L. The experimental study of tumor progression: a review. Cancer Res $1954 ; 14: 327-39$.

10. Foulds L. Tumor progression. Cancer Res 1957 ; 17 : 355-6.

11. Kinzler KW, Vogelstein B. Lessons from hereditary colorectal cancer. Cell 1996 ; 87 : 159-170.

12. Stephens PJ, Greenman CD, Fu B, et al. Massive genomic rearrangement acquired in a single catastrophic event during cancer development. Cell $2011 ; 144: 27-40$.

13. Kandoth C, McLellan MD, Vandin P, et al. Mutational landscape and significance across 12 major cancer types. Nature $2013 ; 502: 333-9$

14. Lawrence MS, Stojanov P, Mermel CH, et al. Discovery and saturation analysis of cancer genes across 21 tumour types. Nature $2014 ; 505: 495-501$.

15. Losman JA, Looper RE, Koivunen $P$, et al. (R)-2-Hydroxyglutarate is sufficient to promote leukemogenesis and its effects are reversible. Science $2013 ; 339: 1621-5$

16. Solary $\varepsilon$. Une approche réductionniste du cancer. Med Sci (Paris) $2014 ; 30: 683-7$.

17. Sonnenschein C, Soto AM. Le cancer et ses gènes insaisissables. Med Sci (Paris) $2014 ; 30: 688$-92.

18. Capp JP. Le rôle des phénomènes aléatoires dans le cancer. Med Sci (Paris) $2014 ; 30: 693-8$.

\section{Autres articles de ce numéro relatifs au colloque}

- Zielinska A, Issad T. La recherche sur le cancer : un champ privilégié pour penser les rapports entre hasard, réductionnisme et holisme. Med Sci (Paris) $2014 ; 30: 675-8$.
- Solary $\varepsilon$. Une approche réductionniste du cancer. Med Sci (Paris) 2014 ; $30: 683-7$.

- Sonnenschein C, Soto AM. Le cancer et ses gènes insaisissables. Med Sci

(Paris) $2014 ; 30: 688-92$.

- Capp JP. Le rôle des phénomènes aléatoires dans le cancer. Med Sci (Paris) $2014 ; 30: 693-8$.

- Kupiec JJ. Comment le hasard intervient-il dans le débat entre holisme et réductionnisme? Conclusion du dossier Cancer/Haredhol. Med Sci (Paris) $2014 ; 30: 699-700$.

\section{POUR EN SAVOIR PLUS (sélection d'articles de $\mathrm{m} / \mathrm{s}$ )}

- Microenvironnements tumoraux : conflictuels et complémentaires.

Med Sci (Paris) $2014 ; 30: 343-466$.

- Fridman WH, Sautès-Fridman C. Le microenvironnement tumoral. Med Sci (Paris) $2014 ; 30: 359-65$.

- Provot S. Contrôle de la croissance et de la dissémination tumorales par le microenvironnement. Med Sci (Paris) 2014 ; 30 : 366-71.

- Pellestor F, Gatinois V, Puechberty J, et al. Le chromothripsis. Med Sci (Paris) $2014 ; 30: 266-73$.

- Reynaud C, Billaud M. La théorie de l'équilibre ponctué. Med Sci (Paris) 2011 ; 27 : 921-3.

- Billaud M. L'hétérogénéité intratumorale. Med Sci (Paris) 2012 ; 28 :

1116-9.

- Stoppa-Lyonnet D, Houdayer C. Séquençage de nouvelle génération en génétique médicale. Med Sci (Paris) 2012 ; 28 : 123-4.

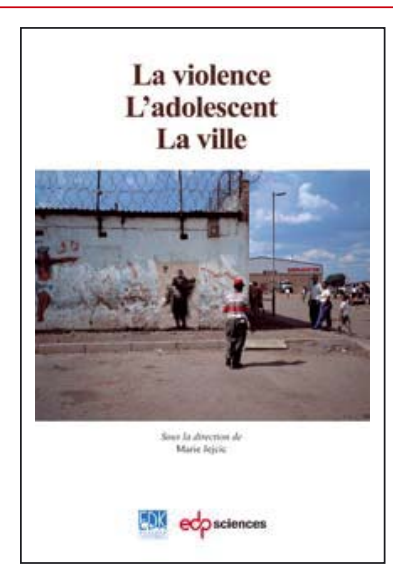

ISBN : 978-2-7598-1138-0 180 pages

\section{TIRÉS À PART}

M. Morange

$-\infty<$

UU À retourner à EDK, 25, rue Daviel - 75013 Paris - Tél. : 0158101905 - Fax : 0143293262 - E-mail : edk@edk.fr

NOM :

Prénom :

Adresse :

Code postal :

a violence s'étend. Comme une mauvaise herbe, elle s'enracine dans nos sociétés européennes avec une sauvagerie nouvelle qui engendre la peur au point de sembler moins l'effet d'une politique locale que d'une civilisation, peut-être même de l'abandon de toute idée de civilisation.

Parce que ce livre est composé à plusieurs voix, il jette un éclairage bref mais précis sur les différentes causes de la violence.

Des auteurs de plusieurs pays et de différents corps de métiers analysent la singularité de la violence selon l'angle où chacun l'observe.

On découvre ainsi que la violence n'est pas monolithique, anonyme, mais qu'elle procède de l'histoire des pays, parfois de l'oubli de leur histoire. Architecte-urbaniste, avocat, journaliste, psychanalystes - psychiatres, psychologues - mais aussi ados d'une de ces banlieues oubliées, aident à préciser les conditions des manifestations de la violence dans la ville, dans la société, dans la clinique et les différentes fonctions qu'elle peut prendre.

Ainsi, ces ados des cités témoignent ici que, convertie, la violence peut aussi devenir vie, énergie et régénérer pour créer, comme ce «polar » qu’ils ont publié alors que brûlaient des autobus...

Pays :

Fonction :

Je souhaite recevoir l'ouvrage La violence - L'adolescent - La ville : $20 €+3 €$ de port $=\mathbf{2 3} €$ TTC

en ................ exemplaire, soit un total de $€$

UU Par chèque, à l'ordre de $\mathbf{E} \mathbf{D} \mathbf{K}$

$\square$ Par carte bancaire : $\square$ Visa $\square$ Eurocard/Mastercard

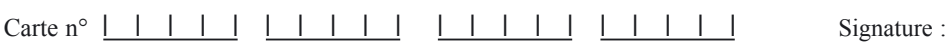

Date d'expiration :

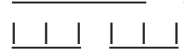

No de contrôle au dos de la carte : 